\title{
A STUDY OF TRMM STATIC EARTH SENSOR PERFORMANCE USING ON-ORBIT SENSOR DATA
}

\author{
Gregory Natanson and Jonathan Glickman \\ Computer Sciences Corporation, 7700 Hubble Drive, Lanham'Seabrook, MD 20706 \\ Phone: 301-794-2151, Fax: 301-794-8355, e-mail: gnatanso a csc.com
}

\begin{abstract}
This paper presents the results of a study of the Bames static Earth sensor assembly (ESA) using on-orbit data collected from the Tropical Rainfall Measuring Mission (TRMM) spacecraft. It is shown that there exist strong correlations between the large penetration angle residuals and the voltages produced by the Offset Radiation Source (ORS). It is conjectured that at certain times in the TRMM orbit the ORS is operating out of its calibrated range, and consequently compts the penetration angle information observed and processed by the ESA. The observed yaw drift between Digital Sun Sensor (DSS) observations is shown to be consistent with predictions by a simple roll-yaw coupling computation. This would explain the large drifts seen on TRMM, where the propagation of the yaw angle between DSS updates does not take into account the possibility of a non-zero roll angle error. Finally, the accuracy of the onboard algorithm used when only three of the four quadrants supply valid penetration angles is assessed. In terms of procedures used to perform this study, the analysis of ESA penetration angle residuals is discovered to be a very useful and insightful tool for assessing the health and functionality of the ESA.
\end{abstract}

\section{INTRODUCTION}

This study was initiated in direct response to several types of anomalous behavior observed on the Tropical Rainfall Measuring Mission (TRMM) since its launch on Thanksgiving Day, 1997. The anomalous behavior includes: (1) occasional pixel jumps observed by TRWM scientists; (2) a region of every orbit in which large penetration angle residuals are observed; (3) occasional occurrences of very large pitch and roll pointing errors during periods of Sun interference in one of the quadrants of the Barnes static Earth sensor assembly (ESA); and (4) larger than desired yaw drifts between yaw updates from the two Digital Sun Sensors (DSSs). Although the spacecraft is still controlled to within the pitch and roll attitude accuracy specification, large pitch and roll pointing errors affect the integrity of one of the science

This paper is declared a work of the U.S. Government and is not subject to copyright protection in the United States. Copyright 02000 The American Institute of Aeronautics and tistronautics Inc. All rights reserved. instruments and, as will be shown in this paper, may be responsible for large drifts in the vaw angle, which occasionally exceed the $3 \sigma$ yaw accuracy requirement.

The TRMM Bames static ESA is composed of four relatively independent quadrants measuring attitude errors about one of the ESA axes." Therefore, opposite quadrants form a redundant pair of attitude sensors, and in principle (as was recently done for AQUA') it is sufficient to process measurements from only one pair of adjacent quadrants. The TRMM attitude control system ${ }^{3}$ takes advantage of this redundancy by using measurements from only three quadrants during quadrant interference by the Sun or the Moon. One of the purposes of this paper is to study the accuracy of this threequadrant algorithm in a more systematic fashion by comparing the resultant roll and pitch pointing errors to those obtained using all four quadrants. The accuracy of the onboard three-quadrant algorithm is also compared with that of a more general algorithm. which can handle the case when only two adjacent quadrants are available.

The paper utilizes a new approach, which analyzes ESA penetration angle residuals. This analysis is found to be a very useful and insightful tool for assessing the health and functionality of the ESA.

\section{MIISSION OUTLINE}

TRMM is an Earth science mission designed to measure the rate of rainfall and the total rainfall occurring over the tropics and subtropics (between the north and south latitudes of 35 degrees). TRMM is a three-axis stabilized, Earth-pointing spacecraft, with the spacecraft's body $z_{B}$ axis directed nominally toward the geodetic nadir. The Earth pointing (rollpitch) is controlled by the attitude control system (ACS) based on measurements taken by a Bames Earth sensor assembly. ${ }^{3.6-6-10}$ (To be accurate, this sensor measures deviations from a "horizon bisector" vector..$^{2.5}$ The difference between the horizon bisector and geodetic nadir vectors is ignored in this paper since it has been shown ${ }^{1,6}$ that this difference is less than 0.01 degree for TRMM.) The yaw angle is updated twice per orbit when the Sun vector crosses the $x_{B} y_{B}$ body plane in the field of view (FOV) of one of the DSSs. It is propagated between the two yaw updates using yaw gyro measurements under the assumption that the $z_{B}$ body axis 


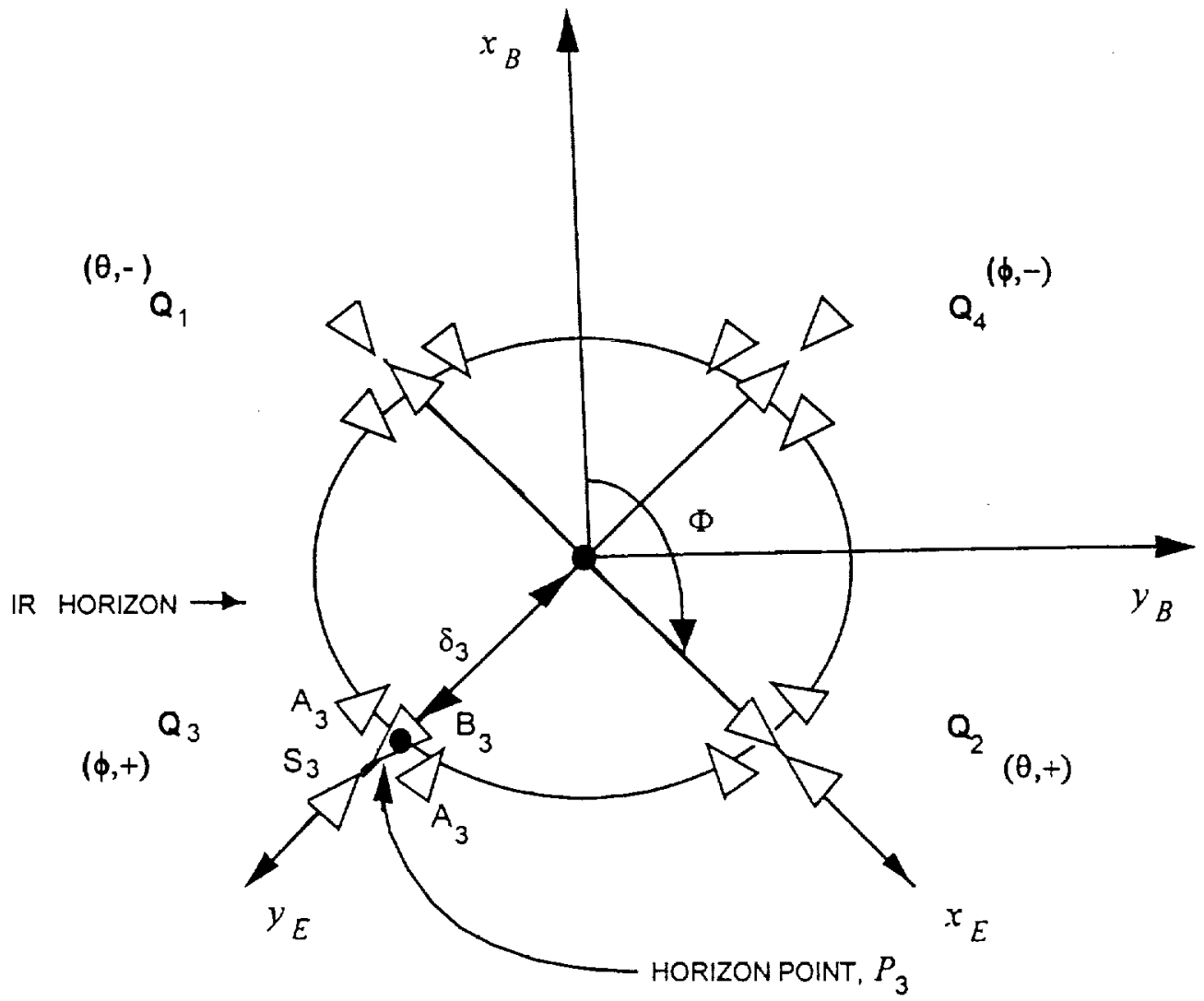

Figure 1. Schematic Configuration of the Static Earth Sensor Assembly

is pointed precisely along the nadir vector.

\section{THE BARNES STATIC ESA}

Operational aspects of the ESA are shown schematically in Fig. I, with the ellipse denoting the Earth infrared horizon. It is composed of four detector assemblies referred to below as quadrants $Q_{1}-Q_{1}$. It is convenient to introduce ${ }^{3,6,8-10}$ the nominal ESA coordinate system, $x_{E} y_{E}$ $z_{E}$, in such a way that opposite quadrants lie in either the $x_{E} z_{E}$ or $y_{E} z_{E}$ plane. Let $x_{B} y_{B} z_{B}$ denote the TRMM body coordinate system (BCS) with $z_{3}$ nominally pointing toward the nadir. The BCS and nominal ESA coordinate systems are related through a rotation $\Phi=135$ degrees about the common $\left(z_{B}=z_{E}\right)$ axis. The $j^{\text {th }}$ quadrant observes the horizon point $\mathrm{P}_{\mathrm{j}}$ with co-elevation $\rho_{j}$ measured from the $z \varepsilon$ axis. It is comprised of four infrared detectors, each with a triangular field of view (FOV) of height 5.2 degrees, two labeled $A_{j}$, and one each labeled $B_{j}$ and $S_{j}$. (For clarity, detectors are labeled only in quadrant $Q_{3}$ of
Fig. 1.) The $A$ and $B$ detectors directly measure the angular penetration of the Earth limb in their FOV, while the $\mathrm{S}$ detector views cold space and is used as a reference. Three raw voltages $-V_{A_{j}}$ (a single signal from both $A_{j}$ detectors), $V_{B . j}$, and $V_{S . j}-$ measured by detectors $A_{j}$, $B_{j}$, and $S_{j}(j=1,2,3$, or 4$)$ are converted by the spacecraft onboard computer (OBC) into a 'penetration angle', $X_{j}$, which is measured from the base of the $B_{j}$ detector to the horizon point $P_{j}$ :

$$
X_{j}=\rho_{j}-\delta_{j}
$$

where the radial mounting angle $\delta_{j}$ is measured from the $z_{\varepsilon}$ axis to the base of the detector $B_{j}$. All the $\delta_{j}$ nominally have the same value, $\delta$, which is a function of the nominal mission altitude $(\delta=70.4$ degrees for TRMM) 
Most of the voltage from each detector comes from the radiation emitted by the detectors to space, so that changes in the horizon image appear as small changes in large voltage outputs. To detect these changes, the outputs from the S-detectors are used to subtract out the signals generated in the horizon viewing detectors. If all detectors were perfectly matched, no other corrections would be necessary. Realistically the match is not perfect, and the so-called Offset Radiation Source (ORS) is used to compensate for heat lost to space. The source replaces the radiation emitted by the detectors to space and thereby reduces the large offset signals. The radiation received from the ORS by each of the detectors should be approximately the same. It has been recognized by Barnes Engineering Company that for different manufacturing reasons the detector outputs cannot be matched to the required accuracy and some additional optical adjustment means are provided. An analysis of TRMM flight data performed below indicates that this optical adjustment is not yet sufficient. As a result, compensation for heat lost to space slightly differs for different quadrants, which results in noticeable pointing errors.

Reference values of penetration angles can be conveniently represented as follows $s^{6.9 .10}$

$$
X_{j}^{\text {ref }} \approx\left\{\begin{array}{ll}
x_{j}^{\text {nom }}-(-1)^{j} \theta, & j=1,2 \\
x_{j}^{\text {nom }}-(-1)^{j} \phi, & j=3,4
\end{array},\right.
$$

where $\theta$ and $\phi$ are Earth pointing errors in the ESA coordinate system,

$$
x_{j}^{\text {nom }} \equiv \rho_{j}^{D}-\delta,
$$

and $\rho_{j}^{D}$ is the nominal angular separation between the geodetic nadir and the boresight of the $\mathrm{j}^{\text {th }}$ quadrant. As initially shown by the author, ${ }^{9,10}$ the oblateness correction to the Earth infrared radius $p_{c}$,

$$
\lrcorner \rho_{j}^{D} \equiv \rho_{j}^{D}-\rho_{c},
$$

can be represented as

$$
\Delta p_{j}^{D} \approx-\frac{1}{4} \alpha \sin ^{2} \lambda \sin \left(2 \rho_{c}\right) \tan ^{2} \rho_{c}+\Delta \rho_{j}^{\hat{\imath}}
$$

where $\alpha$ and $\lambda$ are the Earth flatness coefficient ${ }^{6.11}$ and the spacecraft latitude, respectively, and the quadrantdependent term, $\Delta \rho_{j}^{\uparrow}$, has the form:

$$
\Delta \rho_{j}^{\uparrow} \equiv-\frac{1}{4} \alpha \sin \left(2 \rho_{c}\right) \times\left\{\begin{array}{ll}
N_{E, x}^{2}, j=1,2 \\
N_{E, y}^{2}, j=3,4
\end{array},\right.
$$

where $N_{E, x}$ and $N_{E, y}$ are the projections of the North Pole unit vector $\hat{\mathrm{N}}$ on the axes $x_{E}$ and $y_{E}$.

Under nominal conditions the TRUM velocity vector is either parallel or anti-parallel to the body axis $x_{3}$ (the socalled ' $x$-forward' and ' $x$-forward' modes) so that the oblateness correction (5) takes the form

$$
\begin{array}{ll}
\Delta \rho_{j}^{D} \approx \Delta \rho^{D}-\kappa / 2, & j=1,2 \\
\Delta p_{j}^{D} \approx \Delta p^{D}+\kappa / 2, & j=j, 4
\end{array}
$$

where

$$
d \rho^{D} \equiv-\frac{1}{4} \alpha \sin \left(2 \rho_{c}\right)\left(\tan ^{2} \rho_{c} \sin ^{2} \lambda+\cos ^{2} \lambda\right),
$$

(cf. Eq. (29) in Ref. 11) and $d^{90}$

$$
\kappa \approx \frac{1}{2} \alpha \cos i \cos \zeta \sin \left(2 \rho_{c}\right),
$$

where $i$ is the orbit inclination, and $\zeta$ is the angle between the spacecraft velocity and the North Pole unit vector $\hat{\mathrm{N}}$.

For TRMM, the quadrant-dependent correction (9) does not exceed 0.06 degree and can be neglected when compared with observation errors; ${ }^{12}$ however, for the sake of generality we will keep this term in the following formulas.

When all four quadrants work, Earth pointing errors, $\theta$ and $\phi$, in the ESA coordinate system can be computed using the differences of penetration angles measured by opposite quadrants ${ }^{2.5}$

$$
\begin{aligned}
& \theta \approx\left(X_{1}-X_{2}\right) / 2, \\
& \phi \approx\left(X_{3}-X_{4}\right) / 2 .
\end{aligned}
$$

This explains the choice of the label $\theta$ or $\phi$ for the quadrants $Q_{1}-Q_{+}$in Fig.1. The symbol ' $\rightarrow$ ' or ' - ' near $\theta$ in Fig. 1 indicates whether the measured horizon point is located on the positive or negative side of the $x_{E}$ axis, 
and similarly for $\phi$ and the $y_{E}$ axis.

In principle, any pair of adjacent quadrants can be used to compute $\theta$ and $\phi$. If our knowledge of the Earth infrared radius were precise, each pair of Eqs. (2) would give the same answer as Eqs. (10a) and (10b). Due to errors of various kind, this is not true. One may expect that processing errors would be at least partially eliminated if the pointing errors, $\theta$ and $\phi$, are computed using Eqs. (10a) and (10b), which do not require knowledge of the Earth infrared radius. The latter formulas, however, become inapplicable in the case of quadrant failure, so that one has to use the original formula (2) for the pair containing the failed quadrant. Below we refer to this scheme as the general method (GM) because it applies even if just two adjacent quadrants are working. (Note that the GM always uses Eq. (10a) or (10b) to compute the appropriate pointing error $\theta$ or $\phi$, respectively, for the pair containing two working opposite quadrants.)

In the case of the failure of a single quadrant, there is an interesting alternative, ${ }^{2.3}$ which explicitly exploits the fact that the body $x_{B}$ and $y_{B}$ axes are rotated over the angle of 135 degrees relative to the ESA $x_{E}$ and $y_{E}$ axes. The roll and pitch pointing errors, $r$ and $p$, are proportional to the sum of $\phi$ and $\theta$ and to their difference, respectively,

$$
\begin{aligned}
& r=-\frac{1}{\sqrt{2}}(\phi+\theta), \\
& p \approx \frac{1}{\sqrt{2}}(\phi-\theta) .
\end{aligned}
$$

In the three-quadrant case there are always two pairs of working adjacent quadrants. One of these pairs can thus be used to express $\phi+\theta$ in terms of the difference between two penetration angles, whereas $\phi-\theta$ can be expressed in terms of the difference between penetration angles measured by the second pair of adjacent quadrants. Therefore (depending on which quadrant has failed), roll and pitch pointing errors can be computed by selecting the appropriate formula from each pair of equations:

$$
r=\frac{1}{\sqrt{2}}\left(x_{2}-x_{3}+x\right)
$$

or

$$
r \approx \frac{1}{\sqrt{2}}\left(x_{+}-x_{1}-\kappa\right)
$$

and

$$
p=\frac{1}{\sqrt{2}}\left(x_{3}-x_{1}-x\right)
$$

or

$$
p \approx \frac{1}{\sqrt{2}}\left(X_{2}-X_{4}+k\right)
$$

Eqs. (12), (12'), (13), and (13') are referred to below as the adjacent quadrant method (AQM). It is used by the TRUMM OBC control system ${ }^{5}$ to estimate roll and pitch angles during interference of a single quadrant by the Sun or the Moon. One major advantage of the AQM over the GM is that it does not require knowledge of the Earth infrared radius, and is therefore expected to be more accurate than the direct formulas (2) used by the G.M.

\section{ANALYSIS OF TRMM FLIGHT DATA}

This section describes the results of an analysis performed using the TRMM Ground Attitude Support utilities. ${ }^{3,14}$ The TRMM attitude was estimated using measurements from two DSSs, ESA penetration angles, and gyro data and solving simultaneously for the epoch attitude and gyro biases. Since roll, pitch, and yaw angles are commanded to zero by the TRMM control system, their estimated values are referred to below as pointing errors. In most cases two parallel runs were made using "scalar" and "vector" models for ESA measurements. The loss function of the "scalar" measurement model is constructed using penetration angle residuals for all four quadrants. The "vector" measurement model minimizes the magnitude of the nadir vector residual, and the projections of the vector residuals on the $x_{B}$ and $-y_{B}$ axes are referred to below as pitch and roll measurement errors. (Since the OBC nullifies the observed projections of the nadir vector on these axes, the aforementioned projections of the vector residuals differ only by sign from the corresponding projections of the reference nadir vector. which explains the choice of the axis directions in the definition of the pitch and roll measurement errors.)

Figs. $2 a$ and $2 b$ depict nominal and measured values of penetration angles $\left(X_{j}^{n o m}\right.$ and $X_{j}^{\text {meas }}$, respectively) in the $+x$-forward nominal mode. Note that each pair of opposite quadrants is represented by a single curve in both figures, but for different reasons. The nominal values of penetration angles depicted in Fig. 2a were computed under the assumption that there were no Earth pointing errors, whereas the penetration angles measured by opposite quadrants coincide simply because the TRMM ACS commands the spacecraft attitude to eliminate any differences in penetration angles for each pair of opposite quadrants.

A striking feature of Fig. $2 b$ is a deep minimum near the 


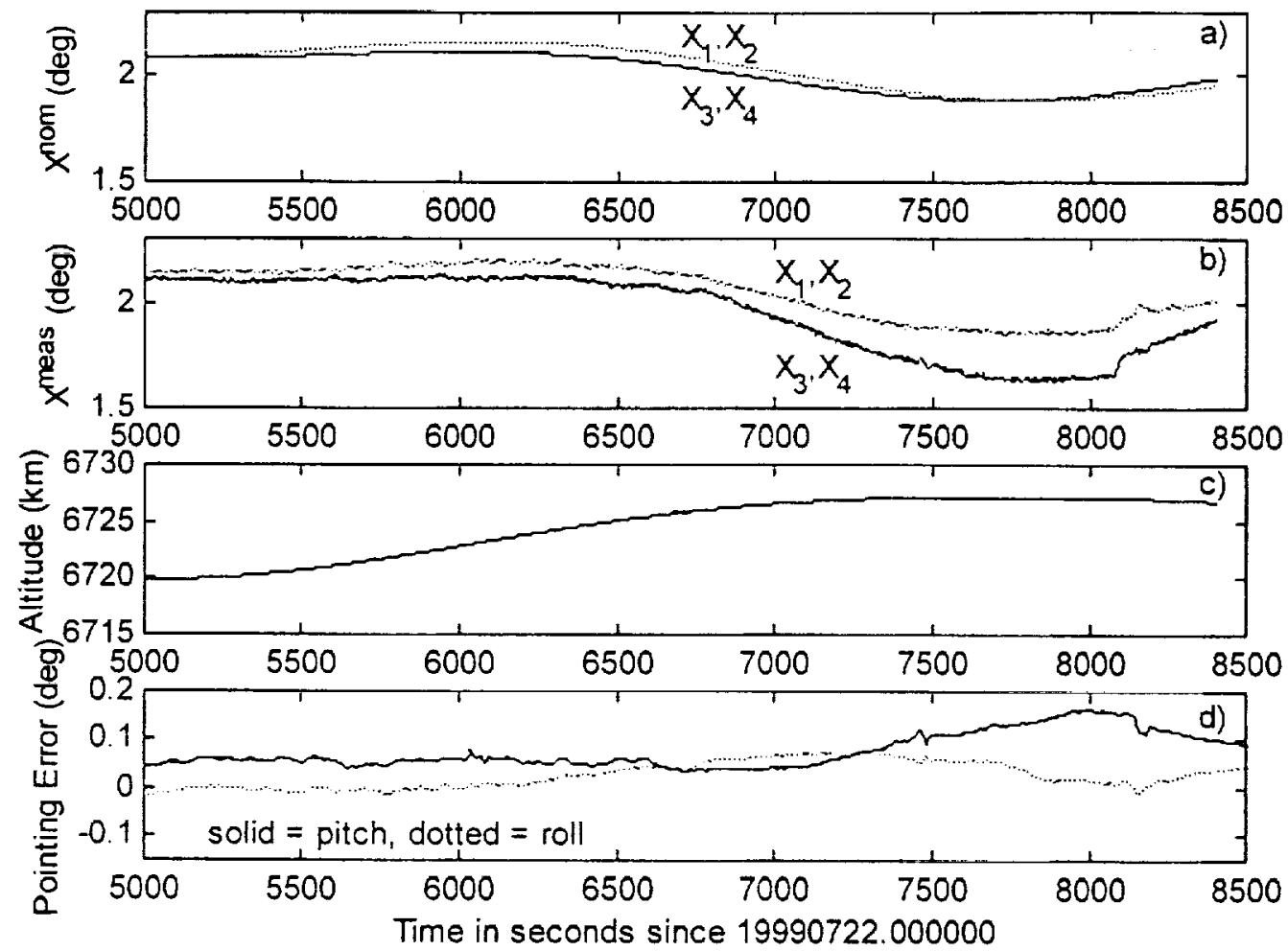

Figure 2. Anomalous Behavior of Measured Penetration Angles Near the Orbit Apogee

orbit apogee (see the altitude plot in Fig. 2c). As one may notice from Fig. 2 a, nominal penetration angles also have smaller values near the apogee (due to a slight decrease in the Earth angular radius $\rho_{c}$ ), but the minimum in Fig. $2 b$ is significantly deeper. Anomalously small values of penetration angles measured by the quadrant $Q_{3}$ are accompanied by noticeable errors in roll and pitch pointing, which implies that the anomalous behavior of penetration angles cannot be attributed to inaccuracies in the Earth modeling. (Remember that both the onboard control and the "vector" measurement model [used to generate ground attitude solutions] utilize Eqs. (10a) and $(10 \mathrm{~b})$ and therefore are unaffected by any errors in the Earth modeling.) We thus conclude that the observed anomalous behavior of penetration angles are caused during the processeing of measurement errors.

The most surprising feature of this anomalous pattern is that penetration angle residuals in the quadrants $Q_{3}$ and $Q_{4}$ increase simultaneously (see Fig. 3a). One would expect that all local changes in the Earth infrared angular radius (such as horizon radiance variations ${ }^{1.2}$ ) should affect only one of the quadrants. There must be a coupling mechanism that simultaneously affects voltage outputs from all four quadrants. The ORS, an onboard heat source that simultaneously compensates all four quadrants for heat lost to space, thus becomes a candidate for the source of this anomaly.

Fig. 3 reveals a pronounced correlation between large penetration angle residuals and ORS voltages. As seen from the figure, the anomalous behavior of penetration angles happens near the maximum of the ORS temperature, which results in a noticeable decrease in the ORS voltage. It is unclear, however, whether the observed errors are directly caused by the increase in the ORS temperature. In fact, both the decrease in the ORS voltage and the accumulation of errors in penetration angles reverse themselves after the spike, which happens 7 minutes before the ORS temperature reaches its maximum. This behavior remains unexplained.

In the example discussed above the anomalous behavior of penetration angles happen to occur near the orbit apogee, but it equally often occurs near the perigee, as illustrated by Fig. 4, which also uses data for the $+x$ - 

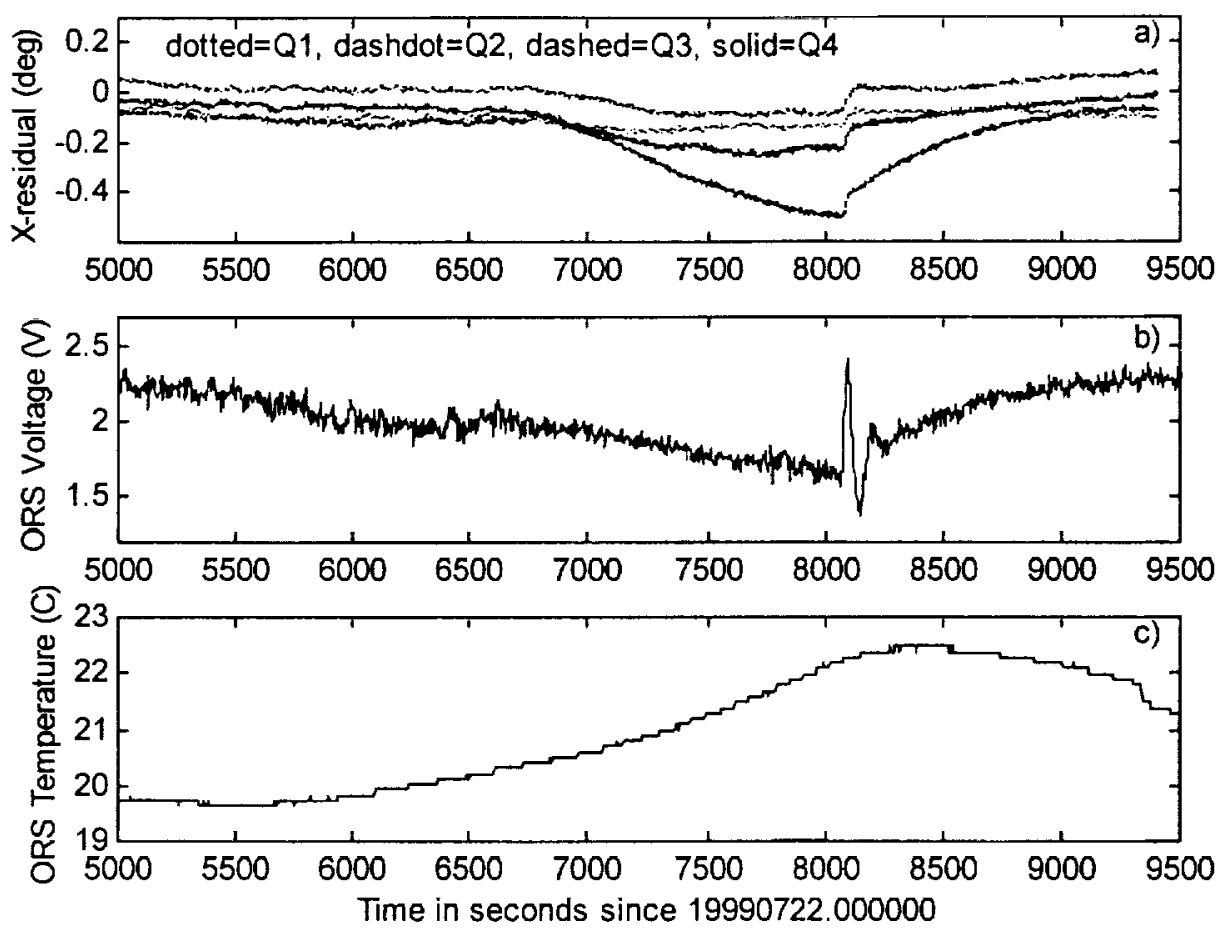

Figure 3. Offset Radiation Source as a Possible Cause for Errors

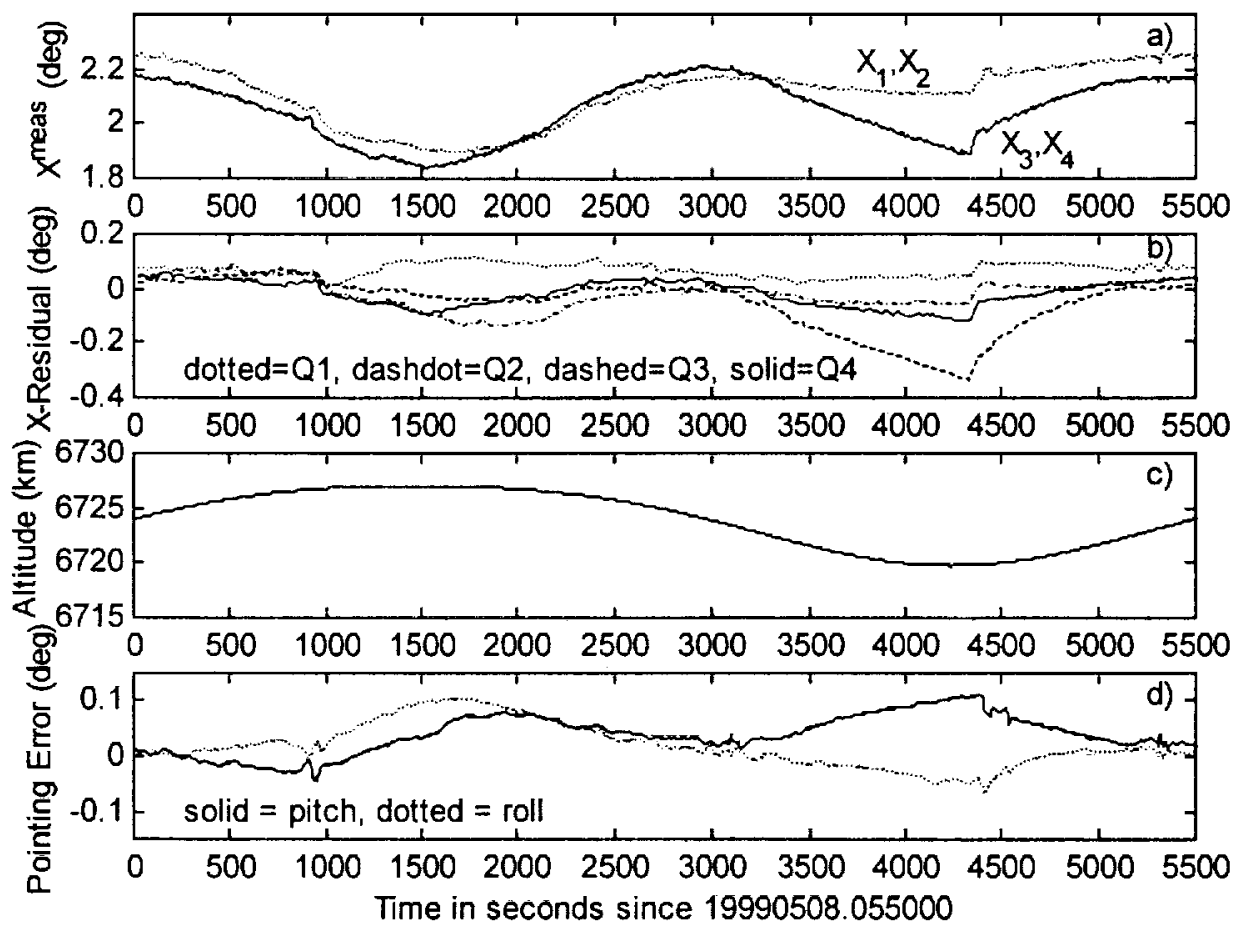

Figure 4. Anomalous Behavior of Measured Penetration Angles Near the Orbit Perigee 


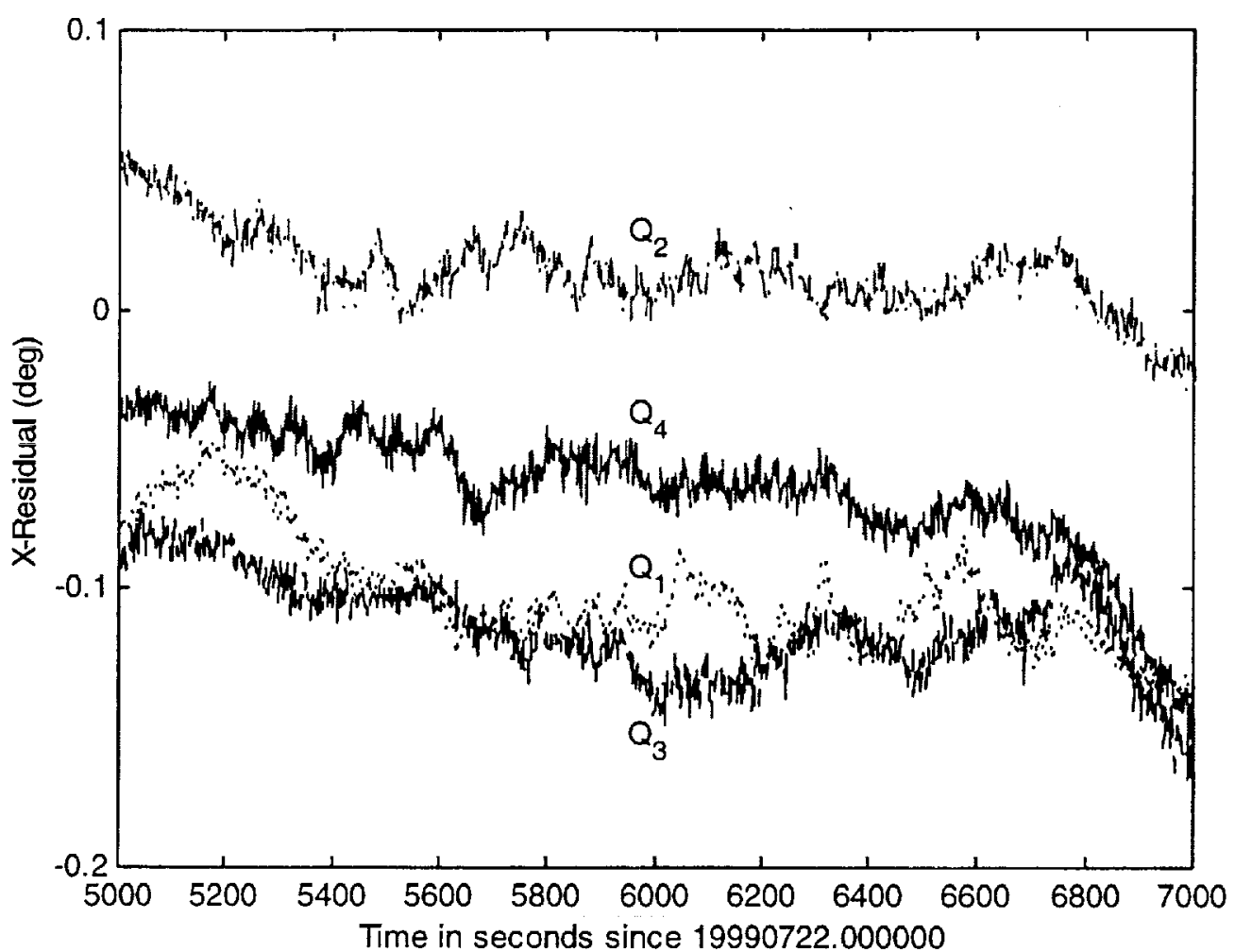

Figure 5. Penetration Angle Residuals for 'Regular' ORS Temperatures

forward mode. The curve representing penetration angles measured by the third quadrant now has the second minimum, which occurs near the apogee and which is again accompanied by relatively large Earth pointing errors.

As seen in Fig. 3, penetration angle residuals start growing when the ORS temperature exceeds $20.5 \mathrm{C}^{\circ}$. For this reason ORS temperatures lying within $20 \pm 0.5 \mathrm{C}^{\circ}$ will be referred below as 'regular'. Fig. 5 magnifies the fragment of Fig. 3a associated with 'regular' ORS temperatures. Note that penetration angle residuals for the quadrants $Q_{1}$ and $Q_{2}$ have opposite sign. As a result, use of three-quadrant formulas (12) and (13') would reduce roll and pitch errors, compared with the conventional four-quadrant algorithm. This is obviously a simple coincidence, but it shows that the Earth modeling is done sufficiently accurate and there is no clear preference between different formulas. In particular, sufficiently accurate estimation of roll and pitch pointing errors can be done using measurements only from two adjacent quadrants.

Finally, let us briefly analyze one of the numerous real- life examples of quadrant interference, when the TRMM control system has to use the $\mathrm{AQM}$ for Earth pointing. Fig. 6 depicts a period in which Q1 experiences Sun interference. The interference period is marked by two vertical dotted lines in Fig. 6b. There is practically no change in the penetration angle residuals (and therefore in pointing errors) immediately after the interference period begins, and where the TRMM onboard control system has stopped processing voltages from the first quadrant and started using the three-quadrant algorithm. Approximately 4 minutes after the beginning of the interference, the ORS voltage rapidly increases, quickly reaching its allowed maximum value of 4.0771 volts. It is saturated for about half a minute, then rapidly decreases, and stays at zero until the end of the interference. Note that pointing errors reach their maximum soon after the ORS voltage is reset to zero and are practically back to their usual range by the end of the interference period. A more detailed analysis is needed to determine the cause of this behavior of the ORS voltage. 

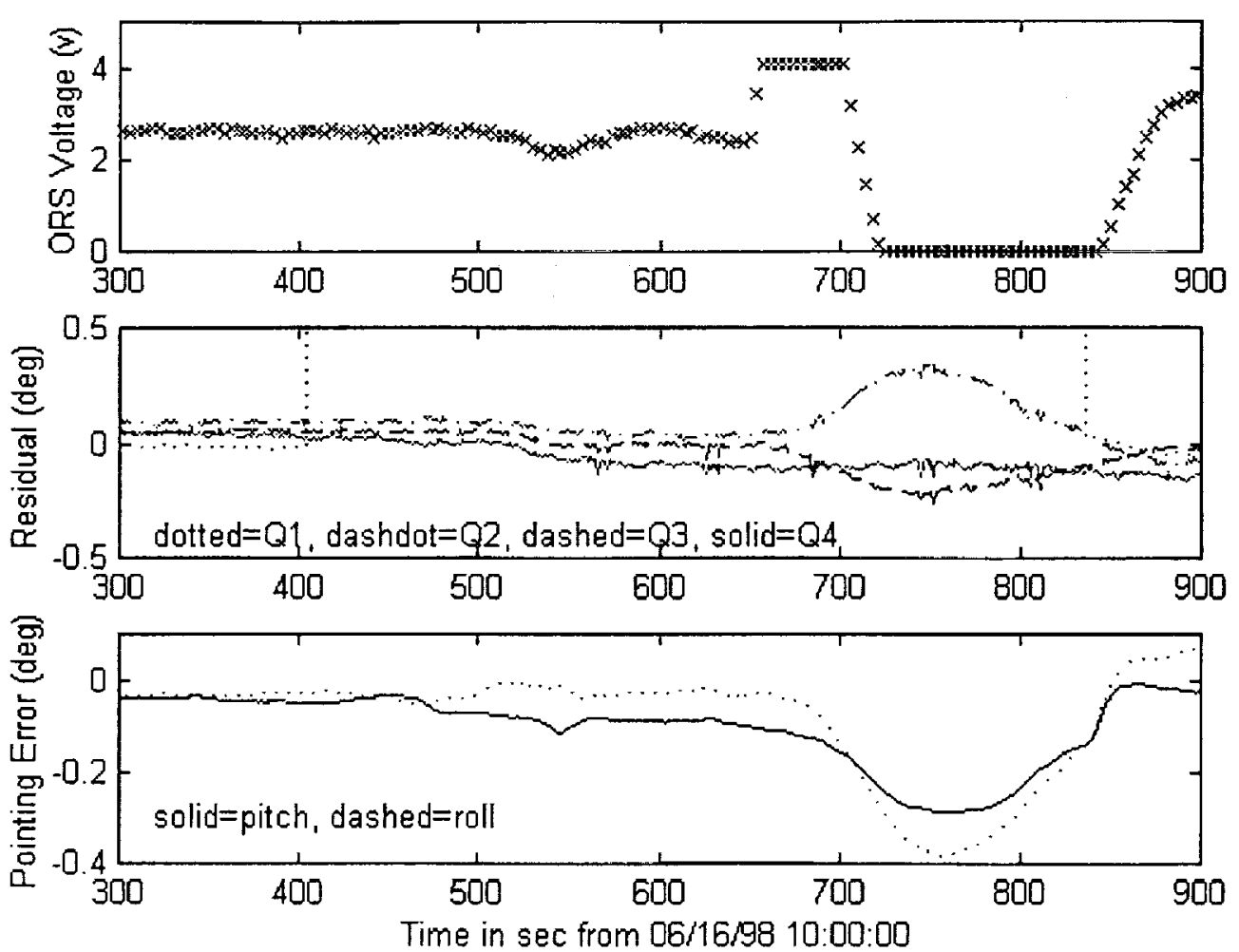

Figure 6. Interference in Quadrant 1

\section{EFFECT OF ROLL POINTING ERRORS} ON TRMM YAW CONTROL

The TRMM $\mathrm{ACS}^{5}$ determines the yaw error by propagating the yaw angle between two DSS yaw updates with the gyro Z-rate. DSS measurements are used only twice per orbit when the Sun vector lies in the $\mathrm{x}_{\mathrm{B}} \mathrm{y}_{\mathrm{B}}$ plane (in the FOV of either the DSS1 or the DSS2). This control law is valid ${ }^{12}$ only under the assumption that there are no Earth pointing errors. To be more precise one needs to compute the $\mathrm{Z}$-component of the angular velocity vector $\bar{\omega}_{\mathrm{R}}$ in the geodetic coordinate system. The latter vector can be evaluated via the equation:

$$
\vec{\omega}_{R} \approx A_{B R}^{T} \bar{\omega}_{B}-\left[0, \omega_{P}, 0\right]^{T} \text {, }
$$

where $A_{B R}$ is the spacecraft attitude relative to the geodetic coordinate system, $\bar{\omega}_{\mathrm{B}}$ are gyro rates, and $\omega_{\mathrm{P}}$ is the pitch rate $( \pm 1 \mathrm{RPO})$. The yaw rate $\dot{\phi}_{\mathrm{Y}} \approx \omega_{\mathrm{R}, \mathrm{Z}}$, corrected for the roll pointing error, $r$, can thus be approximated as

$$
\dot{\phi}_{Y} \approx \omega_{B, Z}+r \omega_{P}
$$

The integral over the first term in the right-hand side of this equation is commanded to zero, so that the yaw pointing error $\phi_{\mathrm{Y}}$ can be represented as

$$
\phi_{Y}(t) \approx \omega_{P} \int_{0}^{t} r\left(t^{\prime}\right) d t^{\prime}
$$

(It is assumed that misalignments of both DSSs have been accounted for and, consequently, that there is no yaw error immediately after the yaw update.)

Fig. 7 compares the estimated yaw errors with their values propagated according to Eq. (16). One can see that propagated yaw errors are in excellent agreement with the estimation results. Thus, it is shown that any roll errors accumulated during ESA processing will contribute 
AIAA-2000-4245

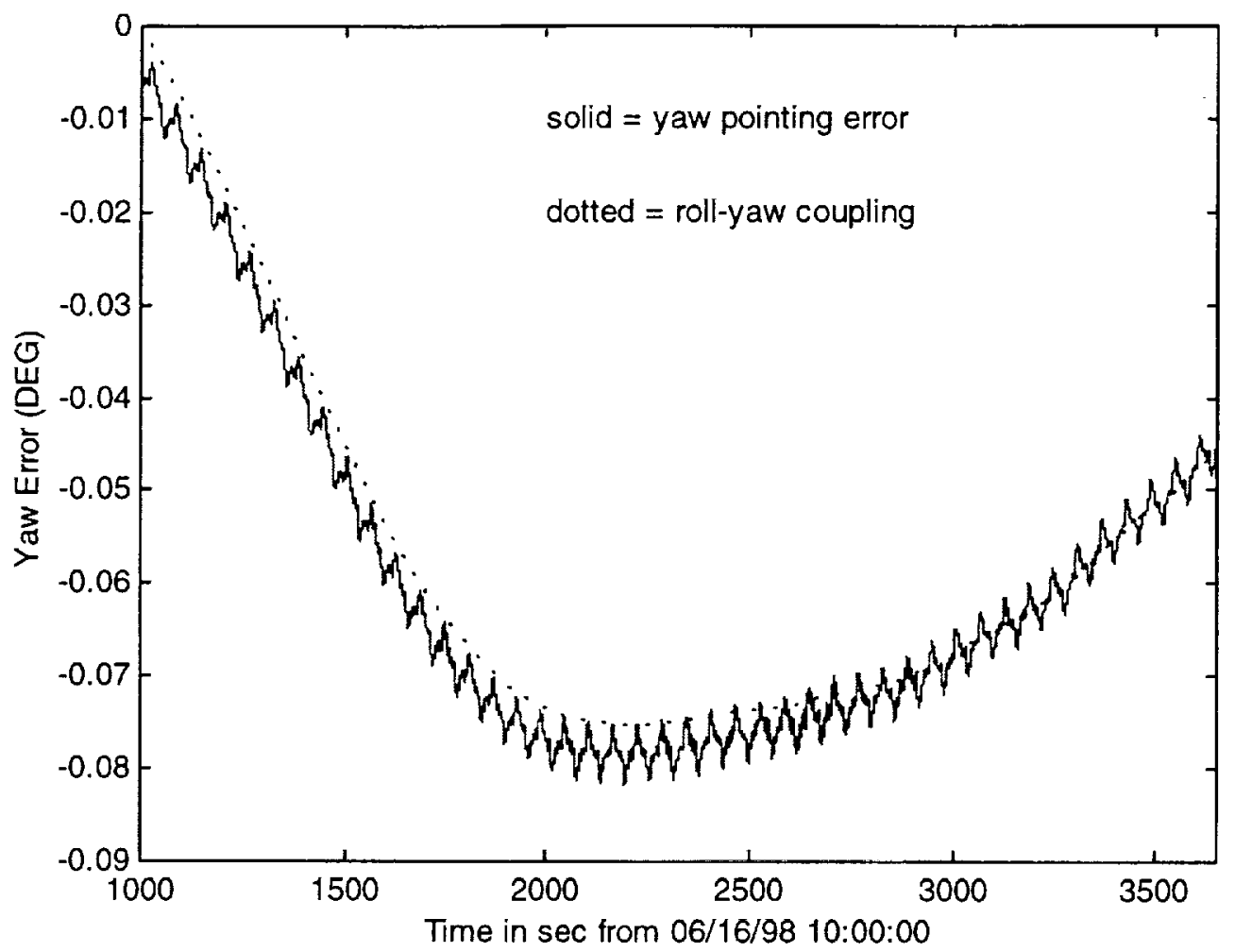

Figure 7. Yaw Errors between DSS1 and DSS2 Yaw Updates

significantly to large drifts in the yaw angle since rollyaw coupling is not taken into account in the onboard propagation equation.

\section{CONCLUSIONS}

The present study reveals a systematic increase in penetration angle residuals during some parts of the TRMM orbit, which results in noticeable pitch and roll pointing errors. It is shown that the errors correlate with anomalous changes in the ORS voltages. This observation points to the ORS as a possible cause for the relatively poor pointing in this region. One advantage of this method is that an analysis of penetration angle residuals would make it possible to identify problems with the ORS for missions not having ORS voltages in telemetry (such as the series of NOAA satellites). Additional study is necessary to confirm this conclusion as well as to find the source of the observed anomalous behavior of the ORS heater. This study may also be important for the NOAA series, for which noticeable image distortions have been reported. ${ }^{15}$

Outside the regions of anomalously large pitch and roll errors, it is found that switching from the four-quadrant algorithm to the three-quadrant algorithm during periods of Sun or Moon interference in one of the ESA quadrants should keep Earth pointing to within the attitude accuracy specification. It is also demonstrated that relatively large roll and pitch pointing errors during interference periods should be attributed to the ORS voltage phenomenon discussed above (rather than to a lower accuracy of the three-quadrant algorithm).

Direct computation of roll-yaw couplings prove that a large error in roll pointing plays a dominant role in the observed large drifts of the yaw angle between yaw updates using DSS measurements. This implies that one can use the spherical Earth approximation, with the only difference being that the infrared horizon, corrected for Earth oblateness, becomes latitude-dependent. ${ }^{\text {II }}$

\section{ACKNOWLEDGEMENTS}

This work was supported by NASA/GSFC, Greenbelt, MD, under Contract GS -35F-4381G, Task Order No. S32415-G. 


\section{REFERENCES}

'J. San; "TRMM ESA On-orbit Anomaly", Technical Memorandurn, NASA, GSFC, Code 572, February 19, 1999.

${ }^{2}$ E. Harvie, O. Filla, and D. Baker, In-Flight Measurement of the National Oceanic and Atmospheric Administration (NOAA)-10 Static Earth Sensor Error, AAS/AIAA Spaceflight Mechanics Meeting, Pasadena, California, February 22-24, 1993

${ }^{3}$ System Error Analysis: Earth Sensor Assembly for the Tropical Rainfall Measuring Mission Observatory, CDRL No. 22B, Revision 2, prepared by EDO Corporation Barnes Engineering Division, NASA Contract No. NAS532463, September 1993

${ }^{4}$ H. D. Schurr, EOS Common Spacecraft Guidance, Navigation, \& Control Subsystem Flight Software Algorithms, No.: D26098, June 30, 1998.

5J. Bracken, J. D'Agostino, and K. Barnes, TRMM ACS Algorithm Document, NASA-GSFC Code, 712.1 document, Build 5.3, November 14, 1996

${ }^{6} \mathrm{M}$. Challa, Attitude Determination Using Static Earth Sensors: Models and Algorithms, 553-FDD94/030R0UD0, prepared by Computer Sciences Corporation, August 1994

${ }^{7}$ J. Keat, M. Challa, D. Tracewell, and K. Galal, Earth Horizon Modeling and Application to Static Earth Sensors on TRMM Spacecraft, Flight Mechanics/ Estimation Theory Symposium, May 16-18, 1995

${ }^{8} \mathrm{G}$. Natanson, Implementation of New Algorithms for the Simulation, Processing, and Calibration of Bames Static Earth Sensor Measurements, Computer Sciences Corporation, Technical Memorandum, November 1996 ${ }^{9} \mathrm{G}$. Natanson, New Algorithms for the Simulation, Processing, and Calibration of Earth Sensor Penetration Angles, Computer Sciences Corporation, Technical Memorandum, November 1996

${ }^{10} \mathrm{G}$. Natanson, Ground-Support Algorithms for Simulation, Processing, and Calibration of Barnes Static Earth Sensor Measurements: Application to the Tropical Rainfall Measuring Mission Laboratory, Proceedings of the Flight Mechanics Symposium, NASA Conference Publication No. 3345, Goddard Space Flight Center, Greenbelt, MD, May 1997

${ }^{1} \mathrm{M}$. Challa and G. Natanson, Recent Developments in Earth Oblateness Modeling for Attitude Determination, Journal of the Brazilian Society of Mechanical Sciences, Vol. 21 (Special Issue: Proceedings of the 14th International Symposium on Space Flight Dynamics), 1999, pp. 134-144

${ }^{12} \mathrm{G}$. Natanson, Accuracy of Earth Pointing Using Adjacent Quadrants of the Barnes Static Earth Sensor: Analysis of Flight Data From the Tropical Rainfall Measuring Mission (TRMM), Technical Memorandum,
CSC-96-968-03, Computer Sciences Corporation, January 2000

${ }^{13} \mathrm{~T}$. W. Flatley, TRMM Yaw, memo from 712.3/TRMM ACS Lead Analyst to Distribution, November 1992

${ }^{14}$ G. Klitsch, M. Lambertson, G. Natanson, R. Strang, et al., Flight Dynamics Distributed Systems (FDDS) Generalized Support Software (GSS) Functional Specification Revision 1, Update 4, CSC/TR92/6023R1UD4, prepared by Computer Sciences Corporation, September 1996

${ }^{15}$ S-Y. Liu, J. Rowe, D. Novak, J. I_M. Chang, Computer Sciences Corporation, Technical Memorandum, July 1998 